\title{
BMJ Open Effect of beta-blocker therapy on clinical outcomes, safety, health-related quality of life and functional capacity in patients with chronic obstructive pulmonary disease (COPD): a protocol for a systematic literature review and meta- analysis with multiple treatment comparison
}

\author{
Claudia Gulea, ${ }^{1}$ Rosita Zakeri, ${ }^{1,2}$ Jennifer K Quint ${ }^{1}$
}

To cite: Gulea C, Zakeri R, Quint JK. Effect of beta-blocker therapy on clinical outcomes, safety, health-related quality of life and functional capacity in patients with chronic obstructive pulmonary disease (COPD): a protocol for a systematic literature review and metaanalysis with multiple treatment comparison. BMJ Open 2018;8:e024736. doi:10.1136/ bmjopen-2018-024736

- Prepublication history and additional material for this paper are available online. To view these files, please visit the journal online (http://dx.doi. org/10.1136/bmjopen-2018024736).

Received 11 June 2018 Revised 28 September 2018 Accepted 4 October 2018

Check for updates

(C) Author(s) (or their employer(s)) 2018. Re-use permitted under CC BY-NC. No commercial re-use. See rights and permissions. Published by BMJ.

For numbered affiliations see end of article.

Correspondence to

Claudia Gulea;

c.gulea18@imperial.ac.uk

\section{ABSTRACT}

Introduction Patients with chronic obstructive pulmonary disease (COPD) who have a clinical indication for beta-blocker therapy, are often not prescribed such medication, despite evidence suggesting that beta-blockers are not associated with adverse respiratory outcomes. The primary objective of this systematic review and meta-analysis is to examine the class effect of beta-blocker use in patients with COPD. We will focus on a broad range of endpoints including, clinical, safety, and patientcentric outcomes such as health related quality of life (HRQoL) and functional capacity. A secondary objective is to explore potential within-class variation in the effects of beta-blockers among patients with COPD, and rank individual agents according to their relative benefit(s).

Methods and analysis MEDLINE, Embase, The Cochrane Library and the Cumulative Index to Nursing and Allied Health Literature (CINAHL) databases will be systematically searched, from inception to present, to identify randomised controlled trials (RCTs) and other prospective and interventional studies of betablocker use in patients with COPD which report on the outcomes of interest. Relative treatment effects with respect to mortality, COPD exacerbations, all-cause hospitalisation, lung function, HRQoL and exercise capacity will be summarised by meta-analysis. Individual treatments (agents) will be compared in a Bayesian network meta-analysis including RCT and observational data, if feasible.

Ethics and dissemination The results of the study will be submitted for publication in a peer-reviewed journal. Only previously published aggregate data will be used for the purpose of this review. PROSPERO registration number CRD42018098983.

\section{Strengths and limitations of this study}

To our knowledge, this is the first review including a quantitative summary of health-related quality of life (HRQoL) outcomes for patients with chronic obstructive pulmonary disease (COPD) who are receiving beta-blocker therapy.

- The systematic review will include evidence from both randomised controlled trial (RCT) and real world (observational) data, in order to extend the generalisability of the findings to patients encountered in clinical practice outside of a tightly controlled RCT environment.

- It may not be possible to conduct a multiple treatment comparison for outcomes that are less frequently reported in our selected patient population (eg, HRQoL, COPD exacerbation rate).

- Subgroup analyses may not be possible due to a lack of data. This may limit the recommendations for specific patient subpopulations (eg, patients with concomitant COPD and coronary artery disease).

\section{BACKGROUND}

Chronic obstructive pulmonary disease (COPD) is a leading cause of death in both the USA and Europe and often coexists with cardiovascular disease (CVD). ${ }^{1}$ Beta-blockers are recommended in several CVD states due to their beneficial effects on mortality and morbidity, as demonstrated in clinical trials including patients with heart failure (HF), ${ }^{2}$ post-myocardial infarction (MI) ${ }^{3}$ and acute coronary syndromes (ACS) ${ }^{4}$

While COPD guidelines $^{5}$ recommend the use of cardioselective beta-blockers, 
patients with concomitant COPD and CVD are often not prescribed these medications due to fear of respiratory deterioration. ${ }^{6}$ Concerns include a beta-blocker induced reduction in patients' forced expiratory volume in the 1st second $\left(\mathrm{FEV}_{1}\right)$ as well as diminished response to the standard COPD therapy (ie, beta-agonists) in the long term. ${ }^{7}$ Although several studies have suggested reduced lung function may be associated with beta-blocker use, tolerability rates among patients with COPD have been good (ie, $>80 \%) .{ }^{8}$ It is therefore unclear whether the observed drop in $\mathrm{FEV}_{1}$ would have a significant impact on longterm outcomes including mortality.

Underutilisation of beta-blockers in patients with COPD has been demonstrated in recent studies of concomitant HF, including a nationwide study from Denmark where only $60 \%$ of patients with comorbid HF and COPD reportedly received a beta-blocker. ${ }^{9}$ A further study in Scotland reported a proportion as low as $18 \% .{ }^{10}$ Studies in other patient populations suggest a significant underuse of betablocker therapy associated with COPD at discharge after ACS, with as many as one-third of patients not receiving the medication. ${ }^{11}$

Contrary to the aforementioned concerns regarding potential risks of beta-blocker therapy in COPD, evidence is accumulating that beta-blockers may importantly confer potential benefits for non-CVD related outcomes in patients with COPD. Two Cochrane reviews reported that cardioselective beta-blockers administered to patients with COPD or mild to moderate reversible airway disease did not exhibit detrimental effects on lung function. ${ }^{12} 13$ Patients with mild or moderate reversible airway disease and COPD had no significant change in their $\mathrm{FEV}_{1}$ after taking beta-blockers when compared with placebo. This effect was significant irrespective of the duration and timing of treatment and beta-blockers were well tolerated in patients with comorbid HF, hypertension or angina. ${ }^{12}$

Observational studies, ${ }^{14}{ }^{15}$ post-hoc analyses from randomised controlled trials (RCTs) ${ }^{16}$ and meta-analyses ${ }^{17}$ have demonstrated that mortality benefits of betablockers extend to patients with COPD and CVD, as well as in subgroups of patients hospitalised for acute exacerbations of COPD. Indeed the primary cause of hospitalisation in COPD is CVD rather than respiratory failure, emphasising the importance of cardiovascular risk management in such patients. ${ }^{18}$ However, other COPD-related outcomes have been less well studied; for instance, little attention has been given to the effect of beta-blockers on rates of acute exacerbation of COPD or patients' functional status which may be more reflective measures of the overall burden of chronic disease and are relevant predictors of hospitalisation. Previous systematic reviews and meta-analyses have focused on short-term findings, particularly when exclusively considering RCT data which is frequently characterised by short follow-up times, ranging from single-dose studies up to 16 weeks' follow-up. ${ }^{12}$

In the present study, we seek to investigate the effects of beta-blocker use on short term (at the end of an RCT or less than 6 weeks-whichever is earliest) and long-term (any time after the end of the intervention) outcomes in patients with COPD, by conducting a systematic literature review (SLR) and quantitative synthesis of contemporary clinical trials and observational data. We will include a broad range of outcomes, including clinical, safety and health-related quality of life (HRQoL) effects, and we will use a meta-analytical approach to demonstrate the class effect of beta-blockers. If feasible, we will perform a network meta-analysis (NMA) to elucidate within-class differences in beta-blocker effects. Demonstration of consistent benefits associated with beta-blocker use in patients with COPD and comorbid CVD, across a range of endpoints, would strengthen the argument in favour of their use in this population.

\section{Rationale for conducting the review}

While there is evidence available to suggest a lack of detrimental effect of beta-blockers in patients with COPD with regards to lung function, previous reviews have only studied cardioselective beta-blockers. In addition, there has been no systematic assessment of the literature regarding the long-term effects of beta-blocker therapy on COPD exacerbation rate, mortality, hospitalisations, HRQoL or functional outcomes in this population. A review by Salpeter et $\mathrm{l}^{13}$ did not include observational data and focused only on $\mathrm{FEV}_{1}$, summarising data published prior to 2010, while Etminan $e t a l^{17}$ investigated mortality alone, but did not quantitatively compare individual betablockers. We will perform an updated review including studies of cardioselective and non-cardioselective betablockers, where the information is available.

Finally, this is also the first systematic review to incorporate both clinical trial and observational data to enable investigation of short-term and long-term outcomes in COPD. If feasible, we will also conduct a NMA comparing the effects of different beta-blockers, including a ranking according to their benefits in patients with COPD.

\section{OBJECTIVES}

We will assess the clinical efficacy (eg, $\mathrm{FEV}_{1}$, rates of exacerbations and mortality), safety (eg, discontinuations), HRQoL (eg, symptom burden) and functional status (eg, exercise capacity) of beta-blockers in patients with COPD from RCTs and observational studies.

We will also determine the effect(s) of individual betablockers on patient prognosis as compared with placebo. If the data permit, we will compare beta-blockers against one another in an NMA for each outcome of interest and explore subgroup effects for specific combinations of COPD and CVD (eg, patients with COPD and concomitant HF, post MI or ACS). Observational data will be integrated in the NMA where feasible.

\section{Rationale for including observational data}

We do not expect to find large RCTs of beta-blockers for some outcomes, ${ }^{19}$ such as COPD exacerbations or 
hospitalisations. Therefore, in order to increase the power and precision of treatment effect estimates, we will include observational studies (prospective and longitudinal). This will allow generalisation of our findings to real-world populations and enable the investigation of longer term outcomes, specifically adverse events which may not be captured in RCTs with short follow-up times.

We will review the degree of bias of observational data and take this into account in our analysis.

\section{AIMS}

1. To identify and critically assess the evidence for beta-blocker use in patients with COPD, with respect to clinical, safety, HRQoL and functional outcomes (SLR and meta-analysis).

2. To compare and contrast within-class effects of beta-blockers in patients with COPD, on clinical, safety, HRQoL and functional outcomes (NMA).

\section{Research questions}

1. What are the beneficial or adverse effects of beta-blocker use in patients with COPD with regard to clinical, safety, HRQoL and functional outcomes? (SLR and meta-analysis)

2. Is there a difference in outcomes between different beta-blockers for patients with COPD? If so, which agents offer the best prognosis for such patients? (NMA)

\section{METHODS}

This study will follow the Preferred Reporting Items for Systematic Reviews and Meta-Analysis guidelines. ${ }^{20}$ Search algorithms will be generated using the population, intervention, control, and outcomes (PICOs) criteria reflecting the research questions.

\section{Population}

Patients with COPD will be defined as those demonstrated by a baseline $\mathrm{FEV}_{1}$ of $<80 \%$ normal predicted value, a $\mathrm{FEV}_{1} / \mathrm{FVC}$ (forced vital capacity) ratio $<70 \%$ consistent with the definition used by the guidelines of the American Thoracic Society ${ }^{21}$ or with a clinical (physician) diagnosis of COPD. Patients will be included if they are aged 35 years old or over. We will exclude patients diagnosed with asthma.

\section{Interventions and comparators}

We will include studies where any of the following betablockers were investigated, whether compared against placebo or another beta-blocker, and given either as a single dose or for an extended period of time:

- Acebutolol, atenolol, betaxolol, bisoprolol, carvedilol, labetalol, metoprolol, nadolol, nebivolol, penbutolol, pindolol, propranolol, sotalol, celiprolol, esmolol, levobunolol, oxprenolol.

Studies investigating palliative care alone or a "watch and wait' intervention will be excluded.
Outcomes

Clinical and safety:

- COPD exacerbations (rate, time to exacerbation).

- All-cause mortality.

- Hospitalisation rate (all cause, and due to COPD exacerbations).

- Lung function $\left(\mathrm{FEV}_{1}\right)$.

- Adverse events (any, including non-specific adverse events, and, discontinuation of beta-blocker therapy due to adverse events).

HRQoL (measured as change from baseline):

- Short form 36, EQ-5D - generic.

- St George's Respiratory Questionnaire for COPD patients.

- Chronic Respiratory Questionnaire.

- COPD Assessment Test.

Functional outcomes (measured as change from baseline):

- Six-minute walk test.

- Incremental shuttle walk test.

Publications investigating in vitro, animal, fetal, molecular, genetic, pathological or pharmacokinetic/pharmacodynamic outcomes without outcomes of interest reported will be excluded.

\section{Study design}

We will include RCT and observational studies (prospective cohort studies), reporting on outcomes of patients with COPD. Studies including a mixed population (eg, COPD and asthma) will be excluded unless they present outcomes separately for the population of interest. Narrative publications, non-systematic reviews, case studies, case reports and editorials will also be excluded.

\section{Search methods and data sources}

Clinical efficacy, safety and HRQoL searches will be conducted in MEDLINE, Embase and Cumulative Index to Nursing and Allied Health Literature via Ovid and The Cochrane Collection Central Register of Clinical Trials with no temporal limits. We will also search ClinicalTrials. gov (www.clinicaltrials.gov). Articles written in languages other than English will be excluded. The search strategy is available in the online supplementary material.

\section{Manual searching}

Reference lists of accepted publications and relevant systematic reviews will be manually searched for additional references.

\section{Selection of eligible studies}

Title and abstract screening

Each title and abstract will be reviewed by two independent investigators to assess eligibility for inclusion in the study according to the predefined inclusion and exclusion criteria. Any disagreements will be resolved through discussion. Where resolution cannot be reached, a third (senior) investigator will make the final decision. For all abstracts deemed eligible for inclusion during the 
first level of review, full-text articles will be retrieved and reviewed.

\section{Full-text screening}

Full papers will be reviewed by two independent investigators. All publications rejected at this stage will be confirmed by a second investigator. For each excluded study, a specific reason for exclusion will be provided and validated by the third investigator. A third investigator will be consulted to resolve any disagreements as necessary.

\section{Patient and public involvement}

As this is a retrospective review of data that has already been collected, patients were not involved in development of the research question or the design of this study at this stage.

\section{Data extraction}

For each included study, data will be extracted on study design, patient characteristics, interventions and outcomes using a Microsoft Excel template developed by the first author with input from the second and third authors. Data elements to be extracted include:

- Study characteristics (country, study design, follow-up time, aims, statistical analysis).

- Population: demographic information (sex, age, ethnicity), sampling methods, inclusion/exclusion criteria, disease severity, comorbidities.

- Interventions and comparators: type of beta-blocker, median, total treatment duration.

- Outcomes: definition of outcome, time point of assessment, value at baseline/time point, change in value from baseline/time point.

Data will be extracted independently by two investigators. Differences in extraction will be resolved through discussion or by a third investigator.

\section{Risk of bias assessment}

The quality of the RCTs will be assessed with the Cochrane tool for assessing risk of bias in RCTs. ${ }^{22}$ Observational studies will be assessed using the Risk Of Bias In Non-randomised Studies - of Interventions (ROBINS-1) tool. ${ }^{22}$ Two investigators will independently complete the appropriate 'Risk of bias' form for each included study. Conflicts will be resolved as described above. Each study will be defined as being at high, low or unclear risk of bias. The quality of evidence contributing to the quantitative analysis will be assessed using Grading of Recommendations, Assessment, Development, and Evaluation ${ }^{23}$ criteria.

\section{Data synthesis}

In order to investigate the clinical, safety HRQoL and functional effects of beta-blocker use in patients with COPD, we will conduct the following analyses:

Meta-analysis of beta-blocker class effect

We will conduct a meta-analysis for each of the outcomes included in the review. For risks, we will extract or manually calculate the incidence and/or prevalence for the

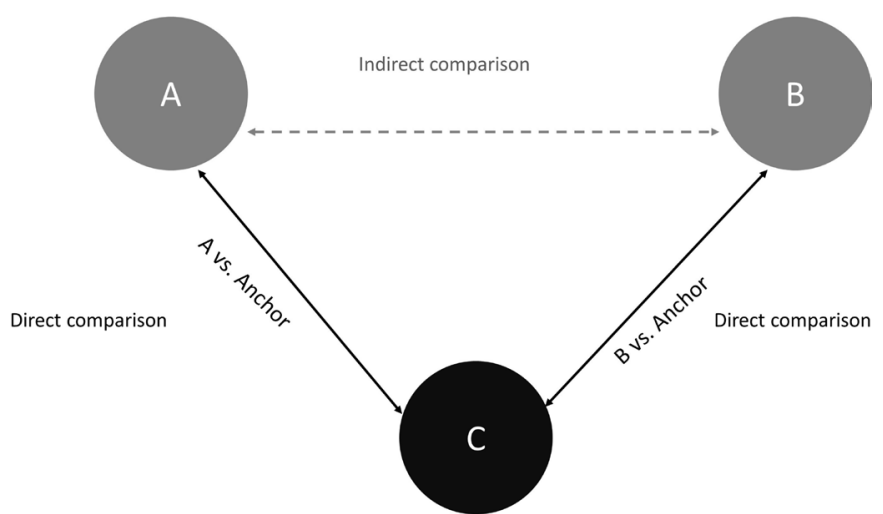

Figure 1 NMA incorporating direct and indirect evidence. NMA, network meta-analysis.

population included in each trial and will meta-analyse relative risks (RR) with $95 \%$ CIs. For continuous variables, we will extract mean differences and 95\% CIs. If clinical homogeneity and the risk of bias are both low, we will pool the results using either fixed-effect or random-effects modelling, depending on the degree of statistical heterogeneity. Higgins and Thompson ${ }^{24}$ suggest heterogeneity is moderate at $\mathrm{I}^{2}$ of $50 \%$, therefore we will consider $\mathrm{I}^{2}>50 \%$ as a cut-off point for the use of a random effects model. If the risk of bias is high or study heterogeneity is high, we will not pool individual studies, but present a narrative synthesis.

\section{NMA of individual beta-blockers}

In the absence of evidence presenting a direct head-tohead comparison of treatments (eg, comparing A with $\mathrm{B})$, an unbiased estimate from an RCT comparing treatments $\mathrm{A}$ and $\mathrm{C}$ and from an RCT comparing $\mathrm{B}$ and $\mathrm{C}$ can be derived in an indirect treatment comparison (NMA) (figure 1). An NMA allows evidence from direct and indirect comparisons to be summarised in a weighted average for all possible comparisons. This analysis will assume that the relative differences between the treatments are exchangeable and apply to all of the included studies.

A feasibility assessment analysing sources of heterogeneity will be conducted on all included studies in the systematic review. This will evaluate whether NMAs can be carried out for the outcomes of interest, by taking into consideration the similarity of patient characteristics, number of studies identified, follow-up times, shape of network and other factors.

For each outcome for which an NMA will be feasible, we will initially include RCT data alone and in a second stage we will add data from propensity-matched or propensity-adjusted studies (ie, observational studies) using Bayesian hierarchical modelling. This is a statistical model which estimates the parameters of the posterior distribution using the Bayesian method. This allows for weighting by study design and provides effect estimates within each study type as well as overall. For example, evidence from RCTs will be first combined to produce estimates; the same will be done for observational studies 
and in a third step, both estimates can be combined to obtain overall results.

While observational data are prone to more bias than RCT data, we believe its inclusion will offset the limitation of RCT data for the analysis of rarely reported outcomes such as quality of life and will help increase generalisability.

We expect little or no RCT data will be available for some outcomes, for example, exacerbation and hospitalisation rates. If this is found to be the case, and where the data permit, we will pool results from included studies, irrespective of study design and perform a meta-analysis of the RR of exacerbation or hospitalisation (due to any reason) secondary to beta-blocker use.

Network maps will be presented to illustrate the treatments that are directly compared against each other and the amount of evidence available for each of the treatments. Separate network maps will be presented for each outcome and per study design.

We will report RRs (and 95\% credible intervals) to compare rates and mean differences for continuous variables. If pooling is possible, we will use OpenBugs V.3.2.3 and R V.3.4.4 software.

\section{Presentation of results}

As data permits, results will be presented for both shortterm and long-term outcomes.

\section{Meta-analysis}

Forest plots will be presented for each outcome of interest. A funnel plot will be constructed to identify evidence of publication bias.

\section{Network meta-analysis}

Results for each endpoint will be presented in league tables for all possible comparisons between treatments of interest along with a pairwise probability (ie, the probability of the treatment being better than a specified comparator).

Forest plots showing the relative treatment effects for each treatment in the network versus the reference treatment (ie, placebo) will be presented.

Surface under the cumulative ranking (SUCRA) diagrams showing the probability that a given treatment ranks first, second, third and so on, among all treatments evaluated in the NMA (with regard to the particular endpoint being considered) will also be presented and should be interpreted alongside the forest plots. ${ }^{25}$ These diagrams will also give the SUCRA percentages of total possible area-under-the-curve when ranking treatments, such that the closer a percentage is to $100 \%$, the higher the treatment ranking is relative to all other treatments.

\section{Subgroup analysis}

Subgroup analyses will be conducted to assess the impact of clinically meaningful treatment modifiers (eg, number and type of comorbidities). The following analyses will be considered, where the data permit: patients with COPD and HF, post-MI, atrial fibrillation, hypertension (or other CVD).

\section{DISCUSSION}

One important limitation of this review is confounding by contraindication. This refers to the situation where a drug is knowingly withheld by a treating clinician due to fears the medication would cause negative effects. In this case, differences in outcomes between treated and untreated patients may be associated with a contraindication for therapy in the untreated patients. This lends itself well to the clinical scenario of beta-blocker administration to patients with COPD since clinicians are hesitant to prescribe the medication. This type of confounding could lead to an underestimation of the RR between those who receive treatment versus those who do not.

However, a key strength of this review is the inclusion of a broad range of outcomes, including quality of life, of relevance to patients with COPD in relation to betablocker use. A quantitative investigation of the trade-off between patient-centric outcomes and clinical and safety effects in this context could contribute new arguments to support the utilisation of beta-blockers in COPD.

\section{Author affiliations \\ ${ }^{1}$ Department of Respiratory Epidemiology, National Heart and Lung Institute, Imperial College London, London, UK \\ ${ }^{2}$ Department of Cardiology, Royal Brompton and Harefield NHS Foundation Trust, London, UK}

Contributors CG is the guarantor. CG drafted the protocol and developed the search strategy. JKQ and RZ advised on inclusion and exclusion criteria and critically reviewed the protocol. All authors read, provided feedback and approved the final manuscript.

Funding CG is funded by a National Heart and Lung Institute PhD studentship.

Competing interests None declared.

Patient consent Not required.

Provenance and peer review Not commissioned; externally peer reviewed.

Open access This is an open access article distributed in accordance with the Creative Commons Attribution Non Commercial (CC BY-NC 4.0) license, which permits others to distribute, remix, adapt, build upon this work non-commercially, and license their derivative works on different terms, provided the original work is properly cited, appropriate credit is given, any changes made indicated, and the use is non-commercial. See: http://creativecommons.org/licenses/by-nc/4.0/.

\section{REFERENCES}

1. Morgan AD, Zakeri R, Quint JK. Defining the relationship between COPD and CVD: what are the implications for clinical practice? Ther Adv Respir Dis 2018;12:175346581775052.

2. Ponikowski P, Voors AA, Anker SD, et al. 2016 ESC Guidelines for the diagnosis and treatment of acute and chronic heart failure: the Task Force for the diagnosis and treatment of acute and chronic heart failure of the European Society of Cardiology (ESC)Developed with the special contribution of the Heart Failure Association (HFA) of the ESC. Eur Heart J 2016;37:2129-200.

3. Sripal Bangalore SP, Messerli FH. How useful are $\beta$-blockers in cardiovascular disease? Anatol J Cardiol 2006;6:358-63.

4. Chatterjee S, Chaudhuri D, Vedanthan R, et al. Early intravenous beta-blockers in patients with acute coronary syndrome-a metaanalysis of randomized trials. Int J Cardiol 2013;168:915-21.

5. Vestbo J, Hurd SS, Agustí AG, et al. Global strategy for the diagnosis, management, and prevention of chronic obstructive 
pulmonary disease: GOLD executive summary. Am J Respir Crit Care Med 2013;187:347-65.

6. Hawkins NM, Virani S, Ceconi C. Heart failure and chronic obstructive pulmonary disease: the challenges facing physicians and health services. Eur Heart J 2013;34:2795-807.

7. Baker JG, Wilcox RG. $\beta$-Blockers, heart disease and COPD: current controversies and uncertainties. Thorax 2017;72:271-6.

8. Kotlyar E, Keogh AM, Macdonald PS, et al. Tolerability of carvedilol in patients with heart failure and concomitant chronic obstructive pulmonary disease or asthma. J Heart Lung Transplant 2002;21:1290-5.

9. Sessa M, Mascolo A, Mortensen RN, et al. Relationship between heart failure, concurrent chronic obstructive pulmonary disease and beta-blocker use: a Danish nationwide cohort study. Eur J Heart Fail 2018;20:548-56.

10. Hawkins NM, Jhund PS, Simpson CR, et al. Primary care burden and treatment of patients with heart failure and chronic obstructive pulmonary disease in Scotland. Eur J Heart Fail 2010;12:17-24.

11. Kratzer L, Noakes $P$, Baumwol J, et al. Under-utilisation of $\beta$-blockers in patients with acute coronary syndrome and comorbid chronic obstructive pulmonary disease. Intern Med J 2018;48:931-6.

12. Salpeter SR, Ormiston TM, Salpeter EE. Cochrane Airways Group. Cardioselective beta-blockers for chronic obstructive pulmonary disease. Cochrane Database Syst Rev 2005;4:CD003566.

13. Salpeter SR, Ormiston TM, Salpeter EE, et al. Cardioselective beta-blockers for reversible airway disease. Cochrane Database of Systematic Reviews 2002.

14. van Gestel YR, Hoeks SE, Sin DD, et al. Impact of cardioselective beta-blockers on mortality in patients with chronic obstructive pulmonary disease and atherosclerosis. Am J Respir Crit Care Med 2008;178:695-700.
15. Gottlieb SS, McCarter RJ, Vogel RA. Effect of beta-blockade on mortality among high-risk and low-risk patients after myocardial infarction. N Engl J Med 1998;339:489-97.

16. Maltais F, Buhl R, Koch A, et al. $\beta$-Blockers in COPD: a cohort study from the TONADO Research Program. Chest 2018;153.

17. Etminan M, Jafari S, Carleton B, et al. Beta-blocker use and COPD mortality: a systematic review and meta-analysis. BMC Pulm Med $2012 ; 12: 48$

18. Sin DD, Anthonisen NR, Soriano JB, et al. Mortality in COPD: Role of comorbidities. Eur Respir J 2006;28:1245-57.

19. Chang CL, Wong $C$, Beckert $L$, et al. $\beta$-blockers in exacerbations of COPD: feasibility of a randomised controlled trial. ERJ Open Res 2017;3:00090-2016

20. Shamseer L, Moher D, Clarke M, et al. Preferred reporting items for systematic review and meta-analysis protocols (PRISMA-P) 2015: elaboration and explanation. BMJ 2015;350:g7647.

21. Qaseem A, Wilt TJ, Weinberger SE, et al. Diagnosis and management of stable chronic obstructive pulmonary disease: a clinical practice guideline update from the American College of Physicians, American College of Chest Physicians, American Thoracic Society, and European Respiratory Society. Ann Intern Med 2011;155:179-91.

22. Higgins JP, Altman DG, Gøtzsche PC, et al. The Cochrane Collaboration's tool for assessing risk of bias in randomised trials. BMJ 2011;343:d5928.

23. Guyatt GH, Oxman AD, Vist G, et al. GRADE guidelines: 4. Rating the quality of evidence-study limitations (risk of bias). J Clin Epidemiol 2011;64:407-15.

24. Higgins JP, Thompson SG. Quantifying heterogeneity in a metaanalysis. Stat Med 2002;21:1539-58.

25. Salanti G, Higgins JP, Ades AE, et al. Evaluation of networks of randomized trials. Stat Methods Med Res 2008;17:279-301. 